\title{
ERJ
}

Engineering Research Journal

Faculty of Engineering

Minoufia University

\section{HEAT CONDUCTION WITHIN ELLIPTIC ENCLOSURE WITH INTERNAL HEAT GENERATION}

\author{
F. M. Mahfouz \\ Department of Mechanical Power, Faculty of \\ Engineering, Menoufia University, Egypt \\ e-mail: fmahfouz64@hotmail.com
}

\begin{abstract}
Exact and numerical solutions for steady heat conduction in the enclosure between two long isothermal elliptic tubes with uniform internal heat generation are obtained. Heat conduction process within the enclosure is mainly influenced by internal heat generation, axis ratio of inner tube and major axes ratio of the two tubes. The solutions are obtained in terms of the temperature and local heat flux distributions. The study has shown that the numerical results are in excellent agreement with the analytical results.
\end{abstract}

Keywords: heat conduction - elliptic tube- axis ratio - heat flux-temperature

\section{Introduction}

Heat transfer within annular enclosures has received considerable attention due to number of engineering applications that include thermal energy storage systems, nuclear reactors, solar energy collectors and some others. In such applications heat energy transfers within the enclosure, that contains initially stagnant fluid, by one or more of the three well known modes of heat transfer. Although free convection is the main mode of heat transfer in most of such systems, the conduction mode of heat transfer is the dominant mode in good number of practical situations. The proper design of such thermal systems necessitates accurate prediction of the heat transfer rate in either mode and also necessitates determination of the condition of mode change. This definitely will depend on the type of fluid, fluid properties, gap geometry and temperature difference $[1,2]$.

Several previous theoretical and experimental studies have investigated pure or coupled modes of heat transfer between isothermal annular enclosures (see for example [3-13]. In case of pure conduction heat transfer in annular enclosures little work has been found. Exact solution exists for conduction between two concentric and eccentric cylinders with internal heat generation in [3]. Exact solution for conduction from two adjacent spheres without heat generation has been obtained in [4] while exact solution for conduction heat transfer between two eccentric spheres with internal heat generation is obtained by Alassar [5]. It seems, as far as this author knows, that the exact solution for heat conduction in the enclosure between two isothermal concentric and confocal elliptic tubes with internal heat generation has not been available so far. Therefore, beside considering the numerical solution, the exact solution of the problem will be obtained. The solution is presented in terms of the temperature and local heat flux distributions along the walls of the enclosure.

The elliptic enclosure geometry can be generated in different ways. In this study the geometry is determined by two parameters which are the axis ratio of the inner tube and the major axes ratio of the two tubes. These two parameters can be varied in the parametric study to cover elliptic enclosures that range between two limiting cases, the first is the enclosure between two concentric tubes while the second is the enclosure between a flat plate and elliptic tube that surrounds it.

\section{Problem Formulation}

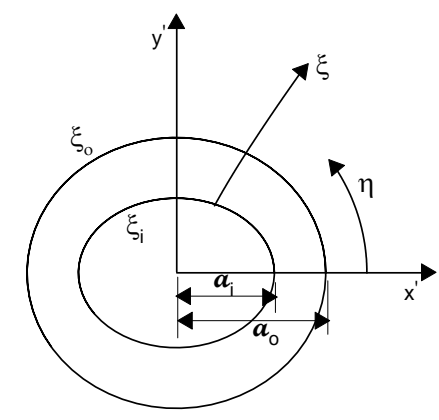

Fig. 1 Physical domain and coordinate system

Consider the annulus between two isothermal, concentric and confocal long elliptic tubes shown in Fig. 1. The heat is generated within the fluid contained in the annulus at steady rate $\mathrm{q}_{\mathrm{v}}$ and then 
transfers within the annulus by conduction and dissipates to the surrounding environment. The temperatures of inner and outer surfaces of the annulus are kept uniform at $\mathrm{T}_{\mathrm{i}}$ and $\mathrm{T}_{\mathrm{o}}$, respectively, with $\mathrm{T}_{\mathrm{i}}>\mathrm{T}_{\mathrm{o}}$. The resulting thermal field would be initially time dependent until it reaches the steady state after a period of time. The steady state solution can be directly obtained by solving the steady state conduction equation. However, this study considers the solution of the time dependent equation as convenient and less expensive route to the steady state solution. Assuming constant physical properties of the fluid in the gap the time dependent conduction equation can be written in Cartesian coordinates as:

$\frac{\partial T}{\partial \tau}=\alpha\left(\frac{\partial^{2} T}{\partial x^{\prime 2}}+\frac{\partial^{2} T}{\partial y^{\prime 2}}\right)+q_{v} / \rho c_{p}$

where $\tau$ is the time, $\mathrm{T}$ is the temperature, $\rho$ is the density, $\mathrm{c}_{\mathrm{p}}$ is the specific heat, $\alpha=k / \rho c_{p}$ is the thermal diffusivity, $k$ is thermal conductivity and $q_{v}$ is the rate of heat generation per unit volume. The above equation can be rewritten in dimensionless form as

$$
\begin{aligned}
& \frac{\partial \phi}{\partial t}=\left(\frac{\partial^{2} \phi}{\partial x^{2}}+\frac{\partial^{2} \phi}{\partial y^{2}}\right)+G \\
& x=x^{\prime} / a_{i}, \quad y=y^{\prime} / a_{i}, \quad t=\tau \alpha / a_{i}^{2}, \\
& \phi=\left(T-T_{o}\right) /\left(T_{i}-T_{o}\right) \text { and } G=\frac{a_{i}^{2} q_{v}}{k\left(T_{i}-T_{o}\right)}
\end{aligned}
$$

Where $\mathrm{G}$ is the heat generation parameter. For proper analytical and numerical treatment of the problem the elliptic coordinates $\xi, \eta$ are adopted. These coordinates are defined as :

$x=\varepsilon_{i} \cosh \xi \cos \eta, \quad y=\varepsilon_{i} \sinh \xi \sin \eta$. Where

$\varepsilon_{i}$ is the eccentricity of inner tube.

In elliptic coordinates equation (2) reads

$$
\frac{\partial \phi}{\partial t}=\frac{1}{J}\left(\frac{\partial^{2} \phi}{\partial \xi^{2}}+\frac{\partial^{2} \phi}{\partial^{2} \eta}\right)+G
$$

Where $J$ is the Jacobian of transformation matrix. In the new coordinates, the steady boundary conditions can be expressed as $\phi=1$ on $\xi=\xi_{i}$ and $\phi=0$ at $\xi=\xi_{o}$ where $\xi_{i}$ defines the inner tube surface $\left(=\tanh ^{-1} \mathrm{Ar}_{\mathrm{i}}\right)$ while $\xi_{o}$ defines the outer tube surface $\left(=\tanh ^{-1} A r_{0}\right), \mathrm{Ar}_{\mathrm{i}}, \mathrm{Ar}_{\mathrm{o}}$, are the axis ratio of the inner and outer tubes, respectively.

\section{Solution procedure}

The solution proceeds by assuming the distribution of dimensionless temperature as an analytical function represented in terms of Fourier series as:

$$
\phi(\xi, \eta, t)=\frac{1}{2} H_{\mathrm{o}}(\xi, t)+\sum_{n=1}^{N} H_{n}(\xi, t) \cos (n \eta)
$$

where $H_{o}$ and $H_{n}$ are the Fourier coefficients and $\mathrm{N}$ is the number of terms considered in the Fourier series. Substitution of $\phi$ defined in eq. (4) in eq. (3) and integrating the equation ( after multiplying by 1 , cos $\mathrm{n} \eta$, one at a time) from 0 to $2 \pi$ results in the following two differential equations:

$$
\begin{gathered}
\frac{\varepsilon_{i}^{2}}{2}\left(\frac{\partial H_{o}}{\partial t} \cosh 2 \xi-\frac{\partial H_{2}}{\partial t}\right)=\frac{\partial^{2} H_{o}}{\partial \xi^{2}}+\varepsilon_{i}^{2} G \cosh (2 \xi) \\
\frac{\varepsilon_{i}^{2}}{2}\left[\frac{\partial H_{n}}{\partial t} \cosh 2 \xi-\frac{1}{2}\left(\delta_{n 2} \frac{\partial H_{o}}{\partial t}+\frac{\partial H_{(n+2)}}{\partial t}+\frac{\partial H_{|n-2|}}{\partial t}\right)\right] \\
=\left(\frac{\partial^{2} H_{n}}{\partial \xi^{2}}-n^{2} H_{n}\right)-\frac{\varepsilon_{i}^{2}}{2} G \delta_{n, 2}
\end{gathered}
$$

Where, $\quad \delta_{\mathrm{n}, 2}= \begin{cases}1 & \text { if } n=2 \\ 0 & \text { if } n \neq 2\end{cases}$

The steady boundary conditions for all Fourier functions presented in equation (5) can be expressed as:

$$
\begin{array}{ll}
\text {-At } \xi=\xi \mathrm{i} & H_{n}=0, H_{o}=2 \\
\text {-At } \xi=\xi_{\mathrm{o}} & H_{n}=0, H_{o}=0
\end{array}
$$

Eqs. 5a,5b along with the boundary condition (6) can now be solved to give $\mathrm{H}_{\mathrm{o}}$ and $\mathrm{H}_{\mathrm{n}}$ which are then used to give the temperature distribution.

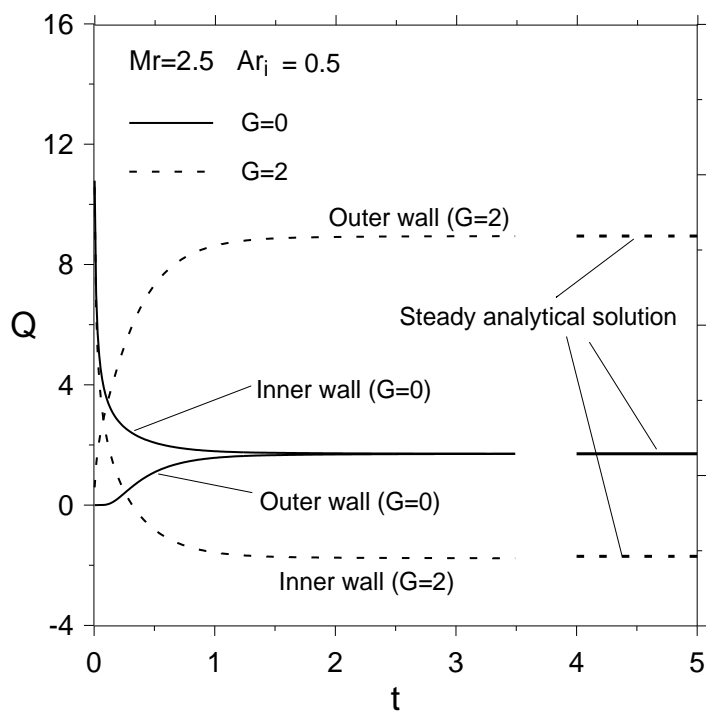

Fig.2 Time variation of numerical dimensionless heat transfer rates from inner and outer walls. 


\section{Numerical solution}

The numerical analysis proceeds further by discretizing equations 5a-b using Crank-Nicolson finite difference scheme. The discretization is carried out over uniform grids in $\xi$ direction and with equal time steps till reaching the steady state solution. The time step is selected very small to ensure the accuracy of the numerical scheme. The resulting tridiagonal system of equations has been solved by TriDiagonal Matrix Algorithm, TDMA

\section{Analytical solution}

Setting the local time derivatives in eqs. $(5 a, 5 b)$ to zero and using boundary conditions (6), the steady state analytical solution of dimensionless temperature distribution is found as :

$$
\begin{gathered}
\phi(\xi, \eta)=-\frac{1}{2}\left(\frac{\varepsilon_{i}^{2}}{4} G \cosh (2 \xi)+c \xi / c_{1}+d\right) \\
+\left[\left(f e^{2 \xi}+g e^{-2 \xi}-\frac{\varepsilon_{i}^{2}}{8} G\right) \cos (2 \eta)\right]
\end{gathered}
$$

Where

$$
\begin{aligned}
& c=\left(2+\frac{\varepsilon_{i}^{2}}{4} G\left(\cosh \left(2 \xi_{i}\right)-\cosh \left(2 \xi_{o}\right)\right)\right. \\
& c_{1}=\left(\tanh ^{-1} A r_{i}-\tanh ^{-1} A r_{o}\right) \\
& d=\frac{\varepsilon_{i}^{2}}{2} G \cosh \left(2 \xi_{o}\right)-c \xi_{o} \\
& f=-g z, g=\frac{\varepsilon_{i}^{2}}{8} G /\left(e^{-2 \xi_{i}}-z e^{2 \xi_{i}}\right), \\
& z=\left(e^{-2 \xi_{o}}-e^{-2 \xi_{i}}\right) /\left(e^{2 \xi_{o}}-e^{2 \xi_{i}}\right)
\end{aligned}
$$

It should be noted the above solution of nonhomogenous PDE (3) is found to be identical to the analytical solution that obtained using Finite Fourier transform associated with Sturm-Liouville system that results from separation of variables to homogeneous form of PDE (3).(see [14])

\section{Heat transfer parameters}

After obtaining the numerical and analytical temperature distributions, the heat transfer characteristics are easily determined. The heat transfer results are presented in terms of dimensionless local heat flux distribution along inner and outer walls and dimensionless total heat transfer rates from inner and outer walls.

The dimensionless local heat flux at the inner and outer walls are defined as :

$$
\begin{gathered}
\bar{q}_{i}=q_{i} P_{i} / \pi k \Delta T_{r e f}, \bar{q}_{o}=q_{o} P_{o} / \pi k \Delta T_{r e f} \\
q_{i}=-k\left(\partial T / \partial S_{n}\right)_{i}, \quad q_{o}=-k\left(\partial T / \partial S_{n}\right)_{o}
\end{gathered}
$$

where $\mathrm{P}_{\mathrm{i}}$ and $\mathrm{P}_{\mathrm{o}}$ are the perimeters of the inner and outer elliptical sections. $S_{n}$ is the local direction of local heat flux normal to the wall. $\Delta T_{r e f}=T_{i}-T_{o}$ is the reference temperature difference. From the above definitions, one can deduce:

$$
\bar{q}_{i}=-\frac{P_{i}}{\pi a_{i}}\left(J^{-1 / 2} \frac{\partial \phi}{\partial \xi}\right)_{i}, \bar{q}_{o}=-\frac{M r P_{o}}{\pi a_{o}}\left(J^{-1 / 2} \frac{\partial \phi}{\partial \xi}\right)_{o}
$$

Defining the dimensionless rate of heat transfer as

$$
Q=\frac{1}{k \pi\left(T_{i}-T_{o}\right)} \int_{0}^{P} q d P, \quad \text { where } \mathrm{P} \text { is the }
$$

perimeter of the elliptic section. The dimensionless rate of heat transfer at inner and outer walls can be

$$
Q_{i}=-\left(\frac{\partial H_{o}}{\partial \xi}\right)_{i} ; \quad Q_{o}=-\left(\frac{\partial H_{o}}{\partial \xi}\right)_{o}
$$
expressed as:

The application of the first law of thermodynamics entails that in the steady state condition: $Q_{o}-Q_{i}=\int q_{v} d V=Q_{t o t}$. It should be noted that the value of $\mathrm{Q}_{0}$ is always positive, meaning that heat transfer dissipates from the outer wall with and without heat generation while the value and sign of $\mathrm{Q}_{\mathrm{i}}$ depends on the heat generation parameter $\mathrm{G}$. The value of $G$ above which the heat dissipates from the inner wall is found from the analytical solution as :

$$
G^{*}=\frac{8 / \varepsilon_{i}^{2}}{2\left(\xi_{i}-\xi_{o}\right) * \sinh \left(2 \xi_{i}\right)-\cosh \left(2 \xi_{i}\right)+\cosh \left(2 \xi_{o}\right)}
$$

The critical value for $G$ as it appears in eq. (10) depends only on the ellipse geometry parameters $\operatorname{Ar}_{\mathrm{i}}$ and $\mathrm{Mr}$, where it increases as the former increases and decreases as the later increases

\section{Results and discussion}

Before proceeding to produce the final results, the numerical and analytical results for steady state solutions of some test cases are obtained and compared with each other and almost identical results are found. Of special interest was the comparison of present results with the well known analytical solution for the case of 1-D heat conduction in cylindrical concentric enclosure with and without heat generation. The comparison has shown that the present analytical results are identical to that of 1-D conduction while the present numerical results shows almost identical results to the known solution of 1-D.

Fig. 2 shows a sample of numerical results in case of no heat generation, $\mathrm{G}=0$ and in case of heat generation at $\mathrm{G}=2$. The figure shows the time variation of heat transfer rates to/from inner and outer walls. In case of $\mathrm{G}=0$ the heat rate from inner wall decreases with time while the heat dissipated from the outer wall increases with time till it almost equals that of inner wall at the steady state. While in 
case of $\mathrm{G}=2$ the steady state heat rate from inner walls is negative, meaning that this heat dissipates from the wall, while the heat that dissipates from the outer wall increases continuously till reaching the steady state value. The figure also shows that the steady state analytical solutions are almost identical to the numerical ones.

Table 1 shows the steady heat transfer rates from the inner and outer walls at $\mathrm{Mr}=2.5$ and $\mathrm{Ar}_{\mathrm{i}}=0.5$ for different values of heat generation parameter, $\mathrm{G}=0$, $0.5,2,5$. The table shows that in case of no heat generation, $\mathrm{G}=0$, the heat transfer rate from the two walls, which in this case is due to temperature difference between the two walls, is positive and equal which means that the heat enters the annulus from inner wall and leaves or dissipates from the outer wall. In case of $\mathrm{G}=0.5$, the heat rates are still positive which means that the heat is still entering the enclosure through the inner wall and dissipates from the outer wall after adding the heat generated in the enclosure. While in cases of $\mathrm{G}=2$ and 5 , the table indicates that the heat is dissipating from the two walls. It can be inferred from these results that there would be a critical value for $\mathrm{G}$ between $0.5<\mathrm{G}^{*}<$ 2 above which heat conduction starts dissipating from the inner wall. This value is obtained from eq. (10) and equal to $G^{*}=0.9809$ for this particular case.

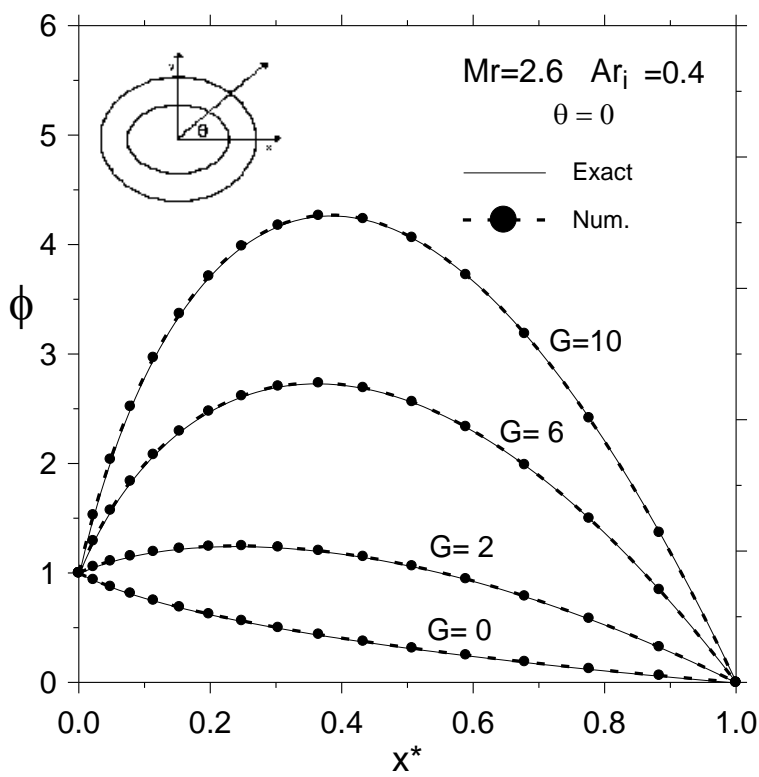

Fig. 3 Distribution of temperature in the gap along major axes at different values of $\mathbf{G}$

Fig. 3 shows the temperature distribution in the annulus gap along the line that coincides with the major axes of the two tubes $(\theta=0)$ for the case of ( $\mathrm{Ar}_{\mathrm{i}}=0.4$ and $\mathrm{Mr}=2.6$ and at different values of $\left.\mathrm{G}\right)$. The figure shows that at $\mathrm{G}=0$ the temperature gradient is negative at the two walls which indicates that local heat enters the enclosure from the inner wall at that point and dissipates from the outer wall. With the presence and increase of internal heat generation parameter $G$ the figure shows that the temperature within the enclosure along major axes increases, attaining maximum value and establishing positive temperature gradient at the inner wall and negative gradient at the outer wall. Such temperature distribution means that the heat flux (and thus heat transfer) dissipates from the two walls. The figure also shows that the position of maximum temperature moves towards the outer wall as $\mathrm{G}$ increases.

a)

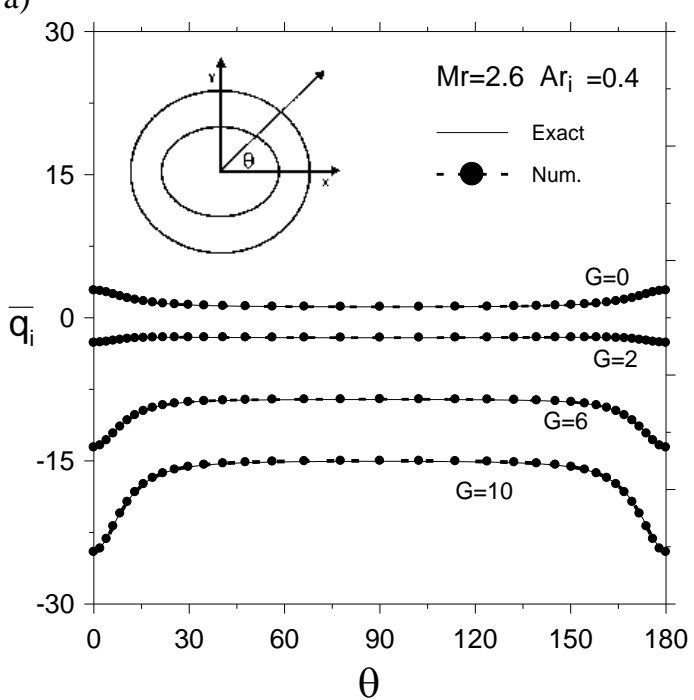

b)

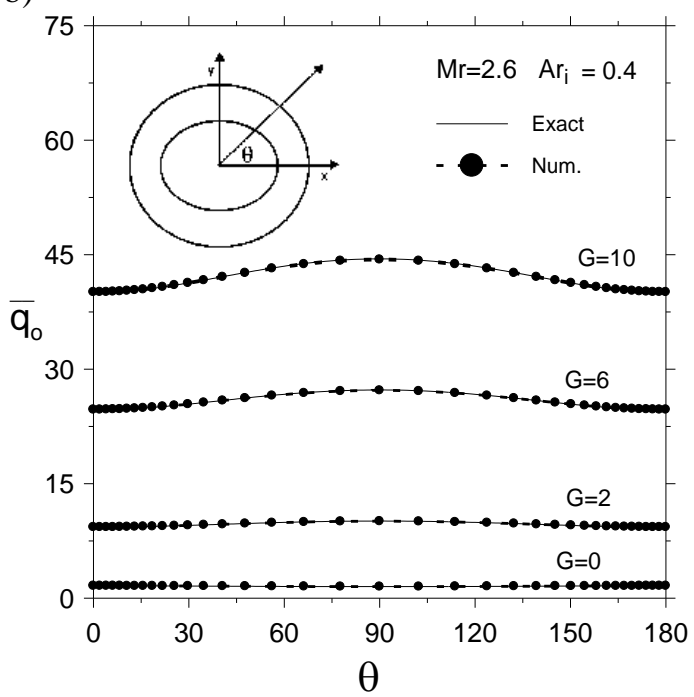

Fig. 4 Heat flux along a) inner wall, b) outer wall at $\mathrm{Mr}=2.6, \mathrm{Ar}_{\mathrm{i}}=0.4$ and different values of $\mathrm{G}$.

Fig. 4 shows the dimensionless heat flux distribution along inner wall (Fig. 4a) and outer wall (Fig. 4b) for the same cases presented in Fig. 3. It can be seen that as $G$ increases the absolute value of heat flux increases at the same point (i. e same $\theta$ ) on the wall. It can be also observed that in case of $\mathrm{G}=0$ the heat flux is positive along the two walls, implying that the heat enters into the enclosure from the inner wall and dissipates from the outer wall. while for $G=2,6,10$ the heat flux is negative along the inner wall but 
positive along the outer wall which means that for these later cases the heat dissipates from the two walls. It can be also inferred from Fig.3 that the critical value for $\mathrm{G}$ lies between $0<\mathrm{G}<2$. This value for this particular case is equal to $G^{*}=0.8445$. Fig. 5 presents the isotherms pattern for the case of $\left(\mathrm{G}=10, \mathrm{Mr}=2.5\right.$ and at different values of $\mathrm{Ar}_{\mathrm{i}}$ ,$\left(\mathrm{Ar}_{\mathrm{i}}=0.005,0.35,0.7,0.998\right)$. The case of $\mathrm{Ar}_{\mathrm{i}}=0.005$ represents the case of an enclosure between a flat plate and the surrounding elliptic tube while the case of $\mathrm{Ar}_{\mathrm{i}}=0.998$ approximates the case of concentric annulus. The figure clearly shows once more that the analytical solution of temperature distribution (right) is almost identical to numerical solution (left). The formation of sub-closed contours is only observed in the wide parts of the annulus near $\theta=90$ while such contours do not appear in the narrow parts near $\theta=0$. The effect of geometry on heat rates $Q_{i}$ and $Q_{o}$ is presented in Tables 2, 3. Table 2 shows the effect of Ari on the rate of heat transfer from the two walls and the total heat transfer rate in case of $\mathrm{G}=10$, $\mathrm{Mr}=2.6$ while Table 3 shows the effect of $\mathrm{Mr}$ on heat rates in case of $\left(\mathrm{G}=5, \mathrm{Ar}_{\mathrm{i}}=0.6\right)$. Presented in the two tables the critical value $\mathrm{G}^{*}$ for each geometry $(\mathrm{Mr}$, Ari). Table 2 shows that for the same $\mathrm{Mr}$ as $\mathrm{Ar}_{\mathrm{i}}$ increases the heat dissipated from the inner wall increases while that from the outer wall decreases and the total heat rate decreases. The increase of $\mathrm{Ar}_{\mathrm{i}}$ at same $\mathrm{Mr}$ decreases the total volume of the enclosure and thus decreases the total heat generation. Table 3 indicates that for the same $\operatorname{Ar}_{\mathrm{i}}$ as $\mathrm{Mr}$ increases the heat dissipated from the two walls increases as well as total heat rate. The increase of $\mathrm{Mr}$ for the same $\mathrm{Ar}_{\mathrm{i}}$ increases the total volume of the enclosure and thus increases the total heat rate.

\section{Conclusion}

Exact and numerical solutions for steady heat conduction in the enclosure between two long isothermal elliptic tubes with uniform internal heat generation are obtained. The solutions are obtained in terms of the temperature and local heat flux distributions. The study has shown that the numerical results are in excellent agreement with the analytical results.

\section{References}

[1] Chiu, C.P., Shich, J.Y. and Chen, W.R., 1999, "Transient natural convection of micropolar fluids in concentric spherical annuli", Acta Mech. 132, pp. 75-92.

[2] Mahony, D.N., Kumar, R. and Bishop, E.H., 1986, "Numerical Investigation of Variable Property Effects on Laminar Natural Convection of Gases Between Two Horizontal Isothermal Concentric Cylinders," ASME J. of heat transfer, 108 , pp. $783-789$.
[3] El-Saden, M.R.,1961, "Heat Conduction in an Eccentrically Hollow, Infinitely Long Cylinder With Internal Heat Generation", J HEAT TRANS-T ASME, 83, pp 510-512.

[4] Alassar, R.S. and Alminshawy, B.J. 2010, "Heat Conduction From two Spheres", AIChE J. 56(9), pp. 2248-2256.

[5] Alassar, R.S, 2011, "Conduction in Eccentric Spherical Annuli”, Int. J. of Heat and Mass Transfer 54 (2011) 3796-3800.

[6] Kuehn T. H., and Goldstein, R. J., 1978, “An Experimental Study of Natural Convection Heat Transfer in Concentric and Eccentric Horizontal Cylindrical Annuli," ASME J. of heat transfer, 100, pp. 635-640.

[7] Farouk, B. and Güçeri, S. I., 1982, "Laminar and Turbulent Natural Convection in the Annulus Between Horizontal Concentric Cylinders," ASME J. of heat transfer, 104, pp. 631-636.

[8] Hessami, M.A., Pollard, A., Rowe, R.D. and Ruth, D.W.,1985,"A Study of Free Convective Heat Transfer in a Horizontal Annulus with a Large Radii Ratio," ASME J. of heat transfer, 107, pp. 603-610.

[9] Pepper, D. W. and Cooper, R. E., 1983, “ Numerical Solution of Natural Convection in Eccentric Annulus," AIAA, 21, pp. 13311337.

[10] Wang S., 1995, “ An Experimental and Numerical Study of Natural Convection Heat Transfer in Horizontal Annuli Between Eccentric Cylinders," J. of Thermal Science, 4(1), pp. 38-43.

[11] Kumar, R., 1988, “ Study of Natural Convection in Horizontal Annuli," Int. J. Heat Mass Transfer, 31 (6), pp. 1137-1148.

[12] Mahfouz, F. M., 2011, "Buoyancy Driven Flow Within an Inclined Elliptic Enclosure" Int. J. of Thermal Sciences, 50, pp. 18871899.

[13] Mahfouz, F. M., 2012, "Heat Convection Within an Eccentric Annulus Heated at Either Constant Wall Temperature or Constant Heat Flux", ASME J. of heat transfer, 134(8), 082502(1-9)

[14] Mahfouz, F. M., 2012, "Natural Convection Within an Eccentric Annulus at Different Orientations", AIAA ,Journal of Thermophysics and Heat Transfer, 26(4), pp. 665-672, 2012.

[15] Donald. W. T., 1990, “ Applied Partial Differential Equations", PWS Publishing Company. 


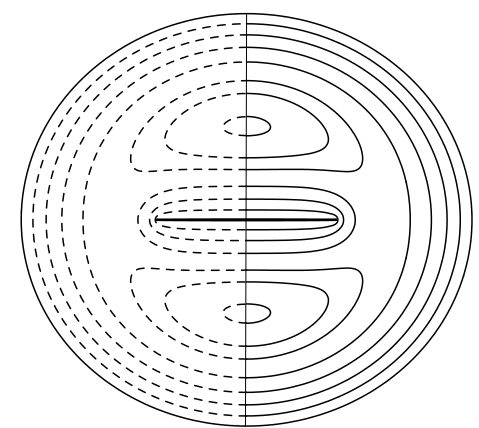

a) $\mathrm{Ar}_{\mathrm{i}}=\mathbf{0 . 0 0 5}$

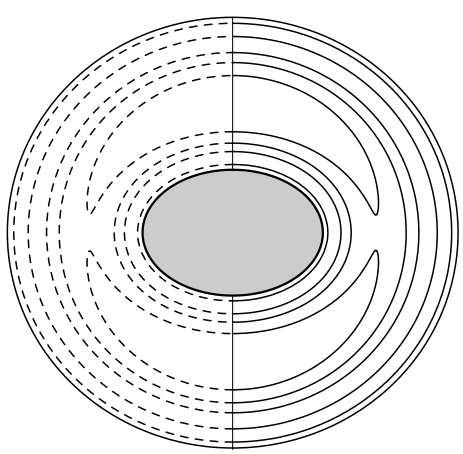

c) $A r_{i}==0.7$

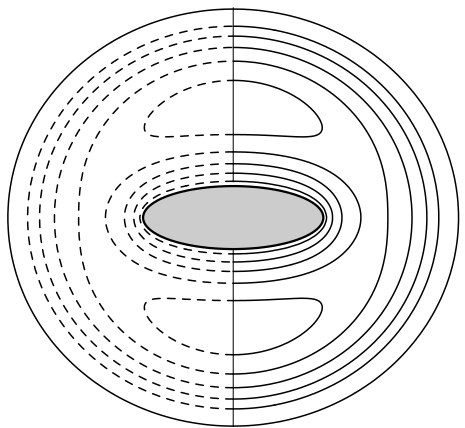

b) $\mathrm{Ar}_{\mathrm{i}}=\mathbf{0 . 3 5}$

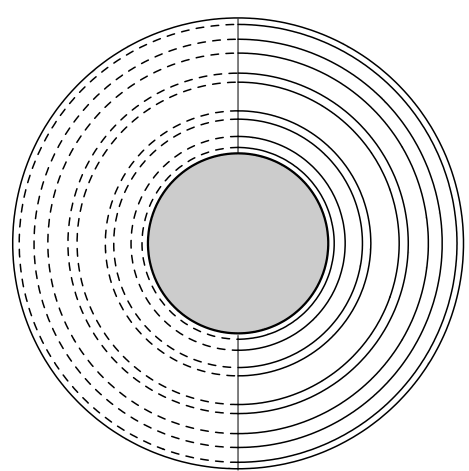

d) $\mathrm{Ar}_{\mathrm{i}}=\mathbf{0 . 9 9 8}$

Fig. 5 Patterns of analytical isotherms (right) and numerical isotherms (left) for the case of $\mathbf{G}=10$, $\mathrm{Mr}=2.5$ and at a) $\mathrm{Ar}_{\mathrm{i}}=\mathbf{0 . 0 0 5}$ (almost flat plate), b) $\left.\mathrm{Ar}_{\mathrm{i}}=0.35, \mathrm{c}\right) \mathrm{Ar}_{\mathrm{i}}=0.7$, and d) $\mathrm{Ar}_{\mathrm{i}}=\mathbf{0 . 9 9 8}$ (almost circular cylinder)

Table 1 Heat rates $Q_{i}$ and $Q_{0}$ at $\left(\mathrm{Ar}_{\mathrm{i}}=0.5 \mathrm{Mr}=2.5\right)$ at different values of $\mathrm{G}$.

\begin{tabular}{|c|c|c|c|c|}
\hline $\mathrm{Q}$ & $\mathrm{G}=0$ & $\mathrm{G}=0.5$ & $\mathrm{G}=2$ & $\mathrm{G}=5$ \\
\hline $\mathrm{Q}_{\mathrm{i}}$ & 1.705 & 0.836 & -1.772 & -6.988 \\
\hline $\mathrm{Q}_{\mathrm{o}}$ & 1.705 & 3.517 & 8.954 & 19.827 \\
\hline $\mathrm{Q}_{\text {tot }}$ & 0 & 2.681 & 10.726 & 26.815 \\
\hline
\end{tabular}

Table 2 Heat rates $Q_{i}$ and $Q_{0}$ at $(G=10, M r=2.6)$ and at different values of $A r_{i}$

\begin{tabular}{|c|c|c|c|c|}
\hline $\mathrm{Q}$ & $\begin{array}{c}\text { Ari }=0.3 \\
\mathrm{G}^{*}=0.808\end{array}$ & $\begin{array}{c}\text { Ari }=0.5 \\
\mathrm{G}^{*}=0.880\end{array}$ & $\begin{array}{c}\text { Ari }=0.7 \\
\mathrm{G}^{*}=0.947\end{array}$ & $\begin{array}{c}\text { Ari }=0.9 \\
\mathrm{G}^{*}=1.099\end{array}$ \\
\hline $\mathrm{Q}_{\mathrm{i}}$ & -16.83 & -17.07 & -17.39 & -17.81 \\
\hline $\mathrm{Q}_{\mathrm{o}}$ & 43.05 & 41.67 & 40.60 & 39.83 \\
\hline $\mathrm{Q}_{\text {tot }}$ & 59.88 & 58.74 & 57.99 & 57.64 \\
\hline
\end{tabular}

Table 3 Heat rates $Q_{i}$ and $Q_{0}$ at $\left(G=5, A r_{i}=0.6\right)$ at different values of $M r$

\begin{tabular}{|c|c|c|c|c|}
\hline $\mathrm{Q}$ & $\mathrm{Mr}=2$ & $\mathrm{Mr}=3$ & $\mathrm{Mr}=4$ & $\mathrm{Mr}=5$ \\
& $\mathrm{G}^{*}=2.049$ & $\mathrm{G}^{*}=0.615$ & $\mathrm{G}^{*}=0.306$ & $\mathrm{G}^{*}=0.183$ \\
\hline $\mathrm{Q}_{\mathrm{i}}$ & -3.295 & -10.809 & -19.197 & -28.761 \\
\hline $\mathrm{Q}_{\mathrm{o}}$ & 12.030 & 29.561 & 56.186 & 91.628 \\
\hline $\mathrm{Q}_{\text {tot }}$ & 15.325 & 30.370 & 75.383 & 120.389 \\
\hline
\end{tabular}

